REVISTA PROYECCIONES: $3-10$.

JORNADAS MATEMATICAS, AGOSTO 1983.DOI: 10.22199/S07160917.1983.0005.00002

\title{
EVOLUCIÓN DEL PENSAMIENTO CUALITATIVO DE MODELOS DEFINIDOS POR ECUACIONES DIFERENCIALES \\ (CHARLA INAUGURAL)
}

\author{
Dr. JORGE BILLEKE GONZALEZ*
}

Los Modelos definidos por ecuaciones diferenciales que nos interesan son aquellos que provienen del mundo de las Ciencias Naturales. Pondremos nuestra atención primeramente en los procesos evolutivos, esto es, procesos cuyos estados cambian con el tiempo. No todo proceso evolutivo es modelizable por ecuaciones diferenciales. Que remos solo los que cumplen las siguientes condiciones

1) Procesos finito dimensionales. Son aquellos cuyos estados E, pueden ser representados, parametrizados por puntos $x=\left(x_{1}, \ldots, x_{n}\right)$ de un abierto de un espacio Euclidiano $\mathbb{R}^{\mathrm{n}}$.

2) Procesos determinísticos. Si $E_{t}$ es la evolución del estado $E$, esta evolución queda determinada, tanto para $t>0$ (futuro) como para $t<0$ (pasado) por el conocimiento de estado presente $E_{0}=E$.

* Profesor Departamento de Matemática y Ciencia de la Computación, Fa cultad de Ciencia. Universidad de Santiago de Chile. 
3) Procesos diferenciables. Si en la parametrización local, la evolu ción $E_{t}$ se expresa por una aplicación

$$
(t, x) \rightarrow p(t, x)
$$

La cual para cada $x$ fijo define una curva en $\mathbb{I}^{n}$ llamada trayectoria. Además $\varphi$ como aplicación de $\mathbb{R} \times \mathbb{R}^{n} \rightarrow \mathbb{R}^{n}$ es continuamente diferenciable.

Juntando coherentemente los abiertos que aparecen en las diversas parametrizaciones obtenemos una variedad diferenciable M llama da Espacio de Estados, Variables internas o Espacio de fases del proceso.

Ejemplo: En la Mecánica Clásica, el péndulo simple tiene por Espacio de Estados $M=S^{\prime} \mathbf{x} \mathbb{R}$ (posiciones $y$ velocidades).

Los objetos matemáticos más usuales en la descripción de los procesos evolutivos son

a) Ecuaciones diferenciales. (Campos Vectoriales, Flujos)

b) Difeornorfismos (Transformaciones puntuales).

Cuando interesa la descripción de un proceso continuo en el tiempo es apropiado considerar un campo diferenciable $\mathrm{x}$ de vectores tangentes a M de modo que las soluciones de

$$
\left\{\begin{array}{l}
\dot{x}=\frac{d x}{d t}=x(x) \\
x(0)=x
\end{array}\right.
$$

que son curvas $\varphi(t, x)=\varphi_{t}(x)$ describa la evolución del estado $x$.

El Campo Vectorial $\mathrm{x}$ describe la evolución infinitesimal de estado

$$
x \stackrel{\text { al estado }}{\longrightarrow} x+\Delta x=x+\Delta t x(x)
$$


Las leyes físicas, biológicas, etc. conocidas de antemano y que rigen el fenómeno determinan el campo vectorial $x$, lo que es equi valente a la ecuación diferencial

$$
\dot{\mathrm{x}}=\mathrm{x}(\mathrm{x})
$$

Ejemplo: Las leyes de Newton de la Mecánica clásica rigen al péndulo simple

$$
\frac{d^{2} \theta}{d t^{2}}+A \operatorname{sen} \theta+B \frac{d \theta}{d t}=0
$$

haciendo $\theta=x_{1}, \dot{\theta}=x_{2}$, tenemos

$$
\left\{\begin{aligned}
& \dot{x}_{1}=x_{2} \\
& \dot{x}_{2}=-A \text { sen } x_{1}-B x_{2}, \text { es decir } x\left(x_{1}, x_{2}\right)= \\
&=\left(x_{2},-A \operatorname{sen} x_{1}-B x_{2}\right)
\end{aligned}\right.
$$

Si solo interesa (o apenas es posible) la descripción de la evolución del sistema en una colección discreta de tiempos $\ldots . t_{-2}, t_{-1}, t_{0}, t_{1}, t_{2} \ldots$ (antes de ayer, ayer, hoy, mañana, pasa do mañana, etc.) estos se pueden identificar con $\mathbf{Z}$.

Entonces es apropiado considerar $f: M \rightarrow M$ diferenciable con inversa $f^{-1}$ también dif., es decir un difeomorfismo, tal que el estado $x_{n+1}$ está dado a partir del estado $x_{n}$ por

$$
x_{n+1}=\varphi\left(x_{n}\right)
$$

Ejemplo: El caso ideal de la población de una colonia de bacterias que crece en proporción geométrica

$$
x_{n+1}=\lambda x_{n}, \lambda>1
$$


La Teoría de Sistemas Dinámicos aborda el estudio cualitati vo de la estructura Global de las órbitas de un sistema definido por campos Vectoriales o Difeomorfismos.

Centremos la atención en esta conferencia en los sistemas de finidos por Ecuaciones diferenciales.

¿Qué caracteriza la conducta cualitativa de un campo de vectores de $\mathbb{R}^{n}$ ?

a) Ubicación y distribución de los puntos de equilibrios, es decir los puntos $x_{0}$ tales que

$$
x\left(x_{0}\right)=0 \quad \text { (singularidades) } p\left(t, x_{0}\right)=x_{0} \quad \forall t
$$

b) Ubicación y distribución de las evoluciones períodicas, es decir órbitas períodicas a $\tau>0$ tq $\varphi(t+\tau, x)=p(t, x) \forall t$

c) Análisis de la conducta asintótica de las órbitas en torno de singularidades y órbitas periódicas.

d) Ubicación y análisis de separatrices.

El primer problema y el mas relevante es de la Estabilidad Estructural es decir la persistencia de las propiedades del modelo por pequeñas perturbaciones de este. ¿Qué significa esto?

Consideremos $\mathrm{X}: \mathbb{R}^{\mathrm{n}} \rightarrow \mathbb{R}^{\mathrm{n}}, \quad \mathrm{Y}: \mathbb{R}^{\mathrm{n}} \rightarrow \mathbb{R}^{\mathrm{n}}$ dos campos de vecto res de clase $C^{k}$, diremos que ellos son $C^{\circ}$-equivalentes si existe $h: \mathbb{R}^{n} \rightarrow \mathbb{R}^{n}$ homeomorfismo que lleva órbitas de $\mathrm{X}$ en órbitas de $\mathrm{Y}$ conservando la orientación de ellas, es decir 


$$
h\left(p_{x}(t, x)\right)=p_{y}\left(t^{\prime}, h(x)\right), t t^{\prime}>0
$$
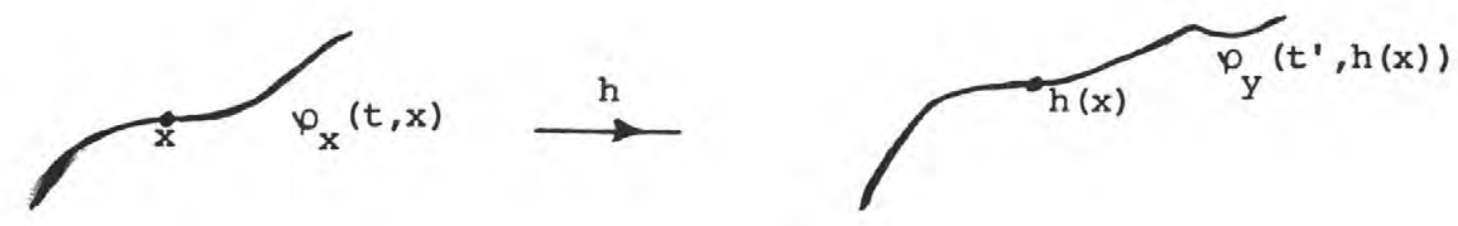

También podemos introducir los términos de $\mathrm{C}^{\circ}$-conjugación $\left(t=t^{\prime}\right) c^{k}$-equiv, $c^{k}-c o n j,\left(h\right.$ es $\left.c^{k}\right)$, linealmente conjugados, Linealmente equivalentes. Si $x^{k}\left(\mathbb{R}^{n}\right)=\left\{\right.$ campos vect. de clase $c^{k}$ en $\left.\mathbb{R}^{n}\right\}$ es un espacio topológico con la topología $C^{r}$.

$x \in x^{k}\left(\mathbb{R}^{n}\right)$ se dice Estructuralmente estable (por $C^{\circ}$-conjugación) si a U C $x^{k}\left(\mathbb{R}^{n}\right)$ abierto, $x \in U$ tal que

$\forall \mathrm{Y} \in \mathrm{U}, \mathrm{Y} \sim \mathrm{X} \mathrm{C}^{\circ}$-conjugado.

Observemos que las conjugaciones preservan singularidades, órbitas periódicas y conductas asintóticas.

La importancia desde un punto de vista práctico de los Siste mas dïnámicos estructuralmente estables radica en que ellos son los llamados a describir cualitativamente los fenómenos observados en el mundo físico, sujetos a inevitables perturbaciones debidas por ejemplo al medio en que estos acontecen o a errores de observación cometi dos al pasar del fenómeno a las ecuaciones Matemáticas. 
Historia: El primero en preocuparse de las ecuaciones diferenciales desde un punto de vista cualitativo fue H. Poincaré en 1881 en su obra "Mémoire sur les courbes définies par une équation differentielle", cu ya problemática es la Mecánica Celeste, en particular la estabilidad del Sistema Solar inspirándose en los trabajos de Newton, Lagrange y Iaplace.

Contemporáneamente a Poincaré, Liapounov, encaró el problema de la estabilidad (pero no de la Estabilidad estructural).

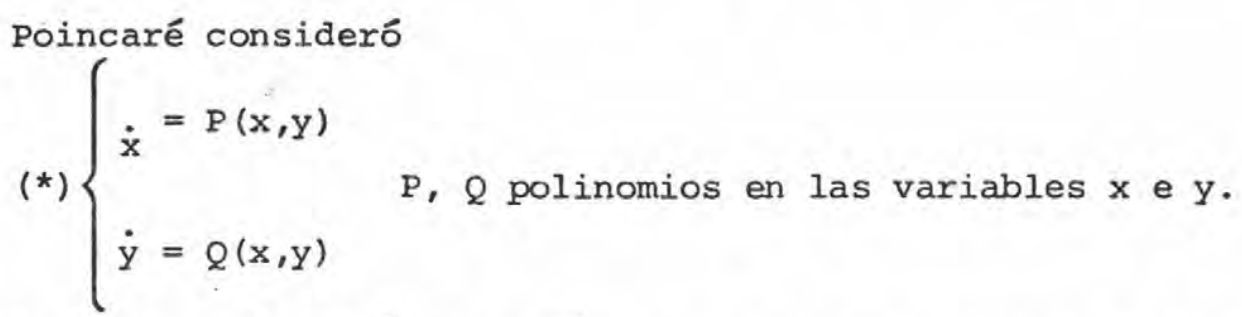

Las Singularidades, $\sum=\mathrm{P}^{-1}(0) \cap \mathrm{Q}^{-1}(0)$, se pueden obtener algebraica mente, lo que no ocurre para las órbitas periódicas, incluso, en el caso $\mathrm{P}$ y $\mathrm{Q}$ de grado 2 no se conoce con exactitud cuántas órbitas periódicas existen y cómo están distribuidas.

El matemático chileno $R$. Bamon ha hecho significativos avances en la respuesta a esta cuestión que está ligada con el 16 problema de Hilbert.

Resultado de Poincaré: Sea $\mathrm{X}=(\mathrm{P}, \mathrm{Q})$ definido por $(*)$. Si $\varphi(t, p)<K$ compacto $\forall t \geqslant 0$ y supongamos que $x$ tenga solo un $n^{\circ} f_{i}$ nito de singularidades (basta que tenga un $\mathrm{n}^{\circ}$ finito de singularidades en $w(p)$, w-límite de $p$, es decir, donde muere la órbita $\rho(t, p)$.

Entonces solo ocurre una de las alternativas siguientes
a) $w(p)=x_{0}, x_{0}$ singularidad
b) $w(p)=\gamma, \gamma$ órbita periódica
c) $w(p)=G, \quad G$ gráfico de separatrices. 
Bendixon en 1905, generalizó este resultado para $\mathrm{P}, \mathrm{Q}$ funcio nes de clase $C^{1}$.

En 1937, Andronov y Pontriagin introdujeron el concepto de Estabilidad estructural y analizaron algunos de ecuaciones diferen ciales en el plano (Ecuaciones Lineales). Estos trabajos fueron conoci dos solo en 1951.

En la década del 60 se produjeron los resultados más importantes en esta Teoría. Veamos algunas definiciones:

Una singularidad $\mathrm{p}$ de $\mathrm{x} \in \mathrm{x}^{\mathrm{k}}\left(\mathbb{R}^{\mathrm{n}}\right)$ se dice hiperbólica si todo autovalor de $\mathrm{DX}(\mathrm{p})$ tiene parte real no nula.

Entonces $\mathbb{R}^{n}=E^{S} \oplus E^{u}$
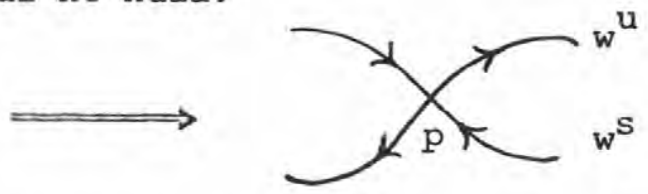

Una órbita $\gamma$ de $x \in x^{k}\left(\mathbb{R}^{n}\right)$ se dice hiperbólica si su trans formación de Poincaré es hiperbólica.

Resultado de Hartmann (1960) Sea p singularidad hiperbólica de $x \in x^{k}\left(\mathbb{R}^{n}\right)$. Entonces $x$ es $C^{\circ}$-conjugado con $D X(p)$ (localmente)

\section{Resultado de Peixoto (1962)}

En toda variedad diferenciable compacta orientable sin borde de dimensión 2, los campos estructuralmente estable son densos. Además Peixoto clasifica tales campos.

Resultado de Kupka-Smale (1962)

Sea M variedad compacta sin borde de dimensión $\mathrm{n}$. Sea $\mathrm{K}-\mathrm{S}$

el conjunto de los campos $\mathrm{x}$ que satisfacen

a) Todas las singularidades y órbitas periódicas de $\mathrm{x}$ son hiperbólicas

b) $\mathrm{w}^{\mathrm{S}}\left(\sigma_{1}\right) \not \mathrm{w}^{\mathrm{u}}\left(\sigma_{2}\right), \sigma_{1}, \sigma_{2}$ elementos críticos de $\mathrm{x}$ (singularidades

y órbitas periódicas).

Entonces $\mathrm{K}-\mathrm{S}$ es residual en $\mathrm{X}(\mathrm{M})$ (Si $M=\mathbb{R}^{n}$ significa abierto $y$ denso). 


\section{Resultado de Morse-Smale (1964)}

Sea $M-S$ el conjunto de los campos $x \in M(M)$ que satisfacen

a) $x$ tiene un número finito de elementos críticos, todos hiperbólicos

b) $\mathrm{W}^{\mathrm{S}}\left(\sigma_{1}\right) \wedge \mathrm{w}^{\mathrm{A}}\left(\sigma_{2}\right), \sigma_{1}, \sigma_{2}$ elementos críticos de $\mathrm{x}$

c) $\Omega(\mathrm{X})=$ el conjunto de puntos no errantes, $\Omega(\mathrm{X})=$ Elementos críticos.

Entonces $M-S$ es un abierto en $*(M)$

Además si M tiene dimensión 2, entonces M-S es además denso y todos los elementos de $\mathrm{M}-\mathrm{S}$ son estructuralmente estables.

Problemas abiertos.

1) ¿será posible encontrar un clase de campos A C $X(M)$ tal que A sea residual $y$ todo $\mathrm{x} \in \mathrm{A}$ sea estructuralmente estable, cualesquiera sea M? No en general, Sí para algunas variedades M.

2) ¿Cuál es la clase más amplia de campos estructuralmente estables?

3) ¿Cómo son los complementos de los K-S?, ¿de los M-S?, etc. 